\title{
Lignolytic-consortium omics analyses reveal novel genomes and pathways involved in lignin modification and valorization
}

Eduardo C. Moraes ${ }^{1 \dagger}$, Thabata M. Alvarez ${ }^{2 \dagger}$, Gabriela F. Persinoti ${ }^{1 \dagger}$, Geizecler Tomazetto ${ }^{1}$, Livia B. Brenelli ${ }^{1}$, Douglas A. A. Paixão', Gabriela C. Ematsu', Juliana A. Aricetti' ', Camila Caldana', Neil Dixon³,

Timothy D. H. Bugg ${ }^{4}$ and Fabio M. Squina ${ }^{5^{*}}$

\begin{abstract}
Background: Lignin is a heterogeneous polymer representing a renewable source of aromatic and phenolic bioderived products for the chemical industry. However, the inherent structural complexity and recalcitrance of lignin makes its conversion into valuable chemicals a challenge. Natural microbial communities produce biocatalysts derived from a large number of microorganisms, including those considered unculturable, which operate synergistically to perform a variety of bioconversion processes. Thus, metagenomic approaches are a powerful tool to reveal novel optimized metabolic pathways for lignin conversion and valorization.

Results: The lignin-degrading consortium (LigMet) was obtained from a sugarcane plantation soil sample. The LigMet taxonomical analyses (based on 16S rRNA) indicated prevalence of Proteobacteria, Actinobacteria and Firmicutes members, including the Alcaligenaceae and Micrococcaceae families, which were enriched in the LigMet compared to sugarcane soil. Analysis of global DNA sequencing revealed around 240,000 gene models, and 65 draft bacterial genomes were predicted. Along with depicting several peroxidases, dye-decolorizing peroxidases, laccases, carbohydrate esterases, and lignocellulosic auxiliary (redox) activities, the major pathways related to aromatic degradation were identified, including benzoate (or methylbenzoate) degradation to catechol (or methylcatechol), catechol orthocleavage, catechol meta-cleavage, and phthalate degradation. A novel Paenarthrobacter strain harboring eight gene clusters related to aromatic degradation was isolated from LigMet and was able to grow on lignin as major carbon source. Furthermore, a recombinant pathway for vanillin production was designed based on novel gene sequences coding for a feruloyl-CoA synthetase and an enoyl-CoA hydratase/aldolase retrieved from the metagenomic data set.

Conclusion: The enrichment protocol described in the present study was successful for a microbial consortium establishment towards the lignin and aromatic metabolism, providing pathways and enzyme sets for synthetic biology engineering approaches. This work represents a pioneering study on lignin conversion and valorization strategies based on metagenomics, revealing several novel lignin conversion enzymes, aromatic-degrading bacterial genomes, and a novel bacterial strain of potential biotechnological interest. The validation of a biosynthetic route for vanillin synthesis confirmed the applicability of the targeted metagenome discovery approach for lignin valorization strategies.
\end{abstract}

Keywords: Lignin, Aromatic compound degradation, Metagenome, Vanillin, Ferulic acid

\footnotetext{
*Correspondence: fabio.squina@prof.uniso.br

${ }^{\dagger}$ Eduardo C. Moraes, Thabata M. Alvarez, and Gabriela F. Persinoti have

contributed equally to this work

${ }^{5}$ Programa de Processos Tecnológicos e Ambientais, Universidade de

Sorocaba, Sorocaba, Brazil

Full list of author information is available at the end of the article
} 


\section{Background}

High global carbon dioxide emission levels and the need for renewable feedstocks for the energy and chemical industries represent great challenges to humankind [1-3]. Lignocellulosic biomass is the most abundant carbon-based material in nature, representing an attractive renewable source to replace oil derivatives and to provide aromatic building blocks for the chemical industry [1-3].

Lignin is one of the major components of plant cell walls, and consists of a highly recalcitrant and heterogeneous polymer network formed via radical coupling reactions involving the three major monolignols: $p$-coumaryl, coniferyl, and sinapyl alcohol, linked by $\mathrm{C}-\mathrm{C}$ and $\mathrm{C}-\mathrm{O}$ bonds [4-6]. The rapid expansion of cellulosic biorefineries has increased the production of lignin-rich streams, which, at present, are mainly burned for energy supply [6]. Controlled deconstruction of the macromolecule to produce lower molecular weight compounds is critical in lignin valorization strategies $[5,7]$. Nevertheless, examples of chemical depolymerization or acidification treatment of lignin to produce low-molecular-weight aromatic compounds have proven to be methods of interest for adding value to lignin streams [8-10]. The depolymerization of lignin into monomers such as guaiacyl, syringyl, vanillin, and syringaldehyde is of commercial interest due to the potential for application of these molecules in the biofuel, food, cosmetic, and other industrial sectors [11].

There is an increasing interest in the development of biological lignin depolymerization routes using enzymes and microorganisms $[12,13]$. In this sense, intensive studies have been performed to elucidate the enzymatic repertoire involved in lignin depolymerization by fungi and bacteria $[13,14]$. Lignin degradation by the white-rot fungi has been extensively studied [15]. However, some bacteria, such as Streptomyces viridosporus T7A, Pseudomonas putida mt-2, Rhodococcus jostii RHA1, Sphingobium sp. SYK-6, and strains of the genera Thauera, Arthrobacter and Rhizobium have also been described in the process of lignin breakdown [16, 17], as well as possessing metabolic pathways for conversion of this complex substrate into valuable commercial products [11]. For example, S. paucimobilis SYK-6 can grow on a variety of lignin-derived compounds and convert them into syringyl or guaiacyl units [18].

Typically, enzyme-based technologies rely on sets of genes produced by a single cultivable organism, whereas natural microbial communities produce biocatalysts derived from a large number of microorganisms, including those considered unculturable, which operate synergistically to perform a variety of bioconversion processes $[19,20]$. Alternatively, the development of enriched microbial consortia has been used as a strategy to select microorganisms with genetic content related to a desirable set of biochemical functions [21-25]. Soil is commonly used as a microbial source for enrichment processes due to its high complexity and countless versatility regarding phenotypic traits, offering enormous potential for establishing microbial communities adapted to a variety of environmental factors, such as $\mathrm{pH}$, temperature, pressure, mineral nutrients, and carbon and energy sources [21-24, 26]. The previous studies reported enriched consortia for degradation of lignocellulosic biomass and aromatic compounds, which based on a combination of culture independent methods (e.g., meta-omics approaches) not only disclosed genes and metabolic pathways involved in this degradation process $[21,24,27]$, but also elucidated the microbial community structures. Although the enrichment process is a powerful tool for development of a community with the desired functions [21, 24, 27], studies involving lignin and ligninderived aromatic degradation have been mostly focused on isolation or culture independent methods $[14,17,18$, $28,29]$.

The present work applied a metagenomic discovery platform, based on targeted community enrichment protocols, to depict bacterial biocatalysts and pathways involved in lignin degradation and aromatic compound conversion (Fig. 1). For this purpose, the lignin-degrading consortium (LigMet) was established using a sugarcane soil sample as inoculum and lignin fragments as the major carbon source in minimal medium. Followed by taxonomical affiliation of microorganism related to lignin degradation in the consortium, metagenomic sequencing allowed for reconstruction of bacterial draft genomes, revealing genes related to lignin depolymerization and metabolic pathways useful in lignin valorization efforts. Moreover, a novel strain of Paenarthrobacter capable of metabolizing lignin fragments and containing several aromatic degradation gene clusters in its genome was retrieved from LigMet. Finally, to validate the usefulness of the metagenomic strategy, the biosynthetic route of vanillin production from ferulic acid was applied using novel enzymes retrieved from LigMet corresponding to feruloyl-CoA synthetase (FerA_B3) and enoyl-coA hydratase/aldolase (FerB_B11).

\section{Methods}

\section{Development of the LigMet}

The sugarcane soil sample was collected from a sugarcane farm in Brazil (GPS coordinates: $-22^{\circ} 04^{\prime} 77.98$, $-48^{\circ} 63^{\prime} 52.99^{\prime \prime}$; Fazenda Tropical). The lignin-degrading consortium (LigMet) was established by adding $1 \mathrm{~g}$ soil to a flask containing $100 \mathrm{ml}$ of culture medium supplemented with a stock solution containing soluble lignin (Fig. 1). The stock solution, referred to herein as lowmolecular-weight (LW) lignin, consists of a supernatant 


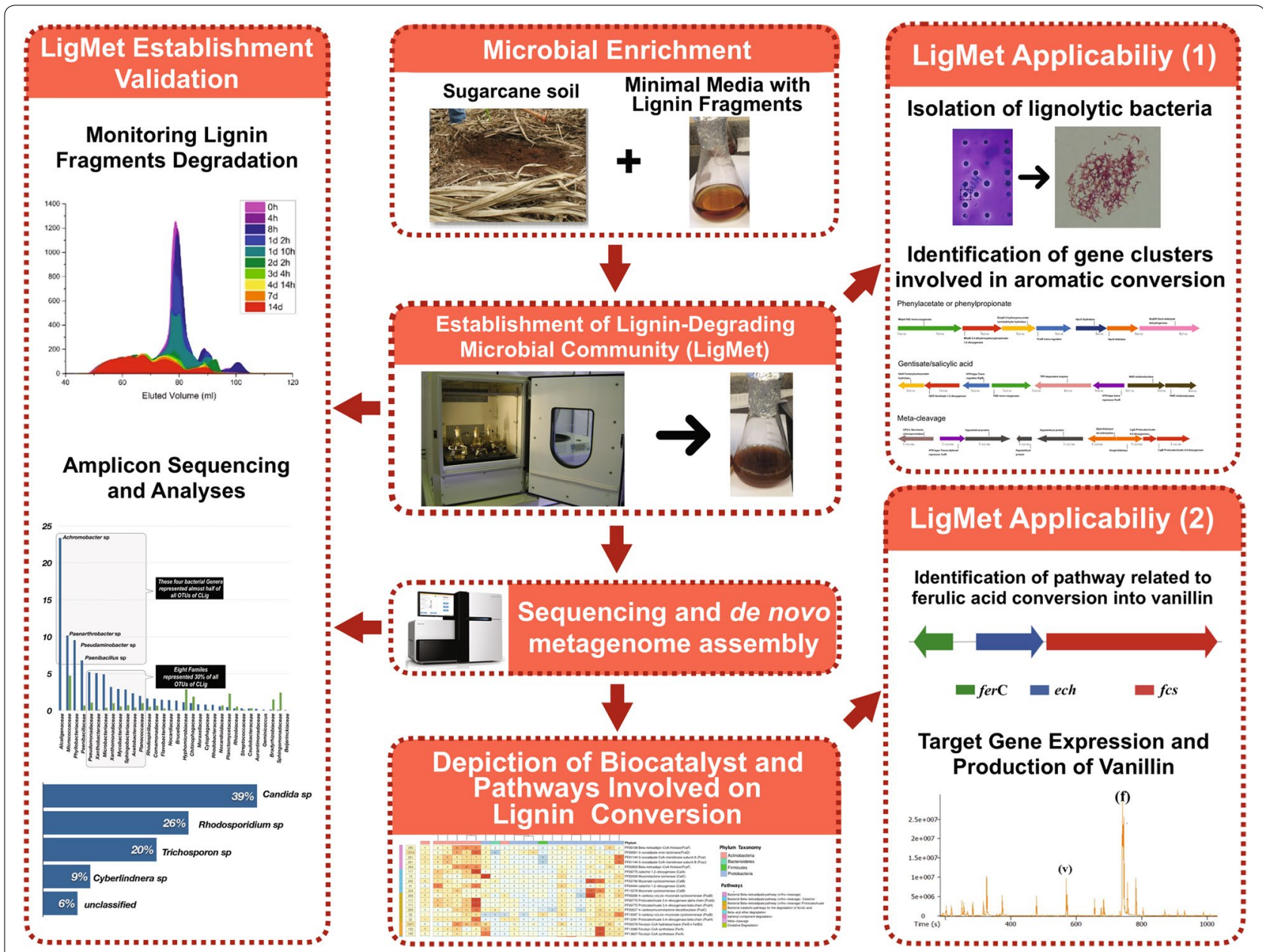

Fig. 1 Schematic representation of the targeted metagenomic discovery platform applied in the present study, including establishment and validation of the lignin-degrading consortium, genetic characterization, and potential applications

obtained after the acidification of black liquor generated from the delignification of steam-exploded sugarcane bagasse [30]. The culture media consisted of a 1:1 dilution of the lignin stock solution in distilled water, with the addition of minimum nutrients from Bushnell Haas Broth (Sigma-Aldrich) and $\mathrm{pH}$ 7.0. The flask was incubated at $30{ }^{\circ} \mathrm{C}$ and $150 \mathrm{rpm}$. Microbial enrichment was obtained by transferring aliquots $(3 \mathrm{ml})$ of the microbial suspension to fresh medium weekly. This procedure was carried out during 50 weeks prior to the first LigMet analysis. The DNS assay [31] was performed to determine the total reducing sugar consumed. LigMet samples were centrifuged and $100 \mu \mathrm{l}$ of the supernatant were mixed to $100 \mu \mathrm{l}$ of the DNS reagent in triplicate as described by the Miller method.

\section{Lignin degradation evaluation}

The consumption of water-soluble lignin fragments present in the stock solution by the microbial community was evaluated by gel permeation chromatography (GPC) in a Superdex ${ }^{\circledR} 30$ prep grade $127 \mathrm{ml}$ column (GE Healthcare) coupled with an ÄKTA System (GE Healthcare). The GPC gradient was performed isocratically using $\mathrm{NaOH} 0.1 \mathrm{~mol} \mathrm{l}^{-1}$ as the eluent at a $0.5 \mathrm{ml} \mathrm{min}^{-1}$ flow rate and $20{ }^{\circ} \mathrm{C}$. For each supernatant, $500 \mu \mathrm{l}$ was diluted in $500 \mu \mathrm{l}$ of $\mathrm{NaOH} 0.1 \mathrm{~mol} \mathrm{l}^{-1}$ prior to injection [8]. The lignin fragments were detected by a UV detector $(280 \mathrm{~nm})$ and the molecular weights were determined using different polyphenolic compounds as external standards.

\section{Sequencing analysis}

Total microbial DNA was extracted using the FastDNA Spin Kit for soil (MP Biomedicals) and purified with Power Clean ${ }^{\circledR}$ DNA Clean-Up Kits (Mo Bio Laboratories) according to the manufacturer's instructions. The hypervariable V4 region of the 16S rRNA and internal transcribed spacer region 2 (ITS2) were amplified using 
universal primers $[32,33]$. PCR products were purified and pooled together in equimolar amounts for sequencing in an Illumina MiSeq system according to the standard procedures. Raw reads were quality assessed using FASTQC (https://www.bioinformatics.babraham.ac.uk/ projects/fastqc/), and preprocessed by removing adapters and low-quality sequences, using Trimmomatic [34]. Quality-filtered reads were merged using Pear [35] and further analyzed using the UPARSE pipeline [36]. Finally, the sequences were clustered into operational taxonomic units (OTU) based on a 97\% identity using the USE$\mathrm{ARCH}$ method. Chimeric sequences were identified and removed using the UCHIME method. The representative sequence from each OTU was assigned to taxonomic taxa using Ribosomal Database Project RDP Classifier [37] and UNITE Fungal ITS training sets [38]. For statistical analyses, the Chao1, ACE, Shannon, and Simpson indices, rarefaction curves, and sample coverage (Good's coverage) were calculated by employing the Phyloseq and Vegan packages using the $\mathrm{R}$ statistical software version 3.2.

For shotgun metagenomic sequencing purposes, $50 \mathrm{ng}$ of the total DNA from LigMet were used to prepare an Illumina Nextera library according to the manufacturer's instructions. The library was validated using an Agilent Bioanalyzer 2100 with the 12000 DNA assay kit (Agilent), quantified by applying the KAPA Biosystem's nextgeneration sequencing library qPCR kit (KAPA), and sequenced on Illumina HiSeq 2500 using a $2 \times 100$ bp paired-end sequencing kit.

Metagenomic reads were quality-filtered using Trimmomatic to remove adaptors and low-quality reads, and then were used for de novo assembly of the metagenome, using IDBA-UD [39]. The assembled contigs were subject to MetaQUAST [40] for quality assessment, and to MetaGeneMark [41] for prokaryotic gene calling, using default parameters. In addition, the contigs were mapped against GenBank NT database, using megablast, and NCBI taxonomic ranks assigned using Blobtools [42], by best sum method. MetaGeneMark was applied, since we were not able to detect eukaryotic sequences in the metagenome assembly. Predicted proteins from LigMet were annotated based on MALT (https://ab.inf.uni-tuebingen.de/ software/malt) searches against SwissProt and UniRef90 databases, and were further compared to the Pfam [43], dbCAN [44], and EggNOG databases [45], using the HMMER3 (http://hmmer.janelia.org/) package. A metabolic pathways analysis was performed using the KEGG web application GhostKoala [46].

Metagenomic contigs were submitted to binning by coverage and sequence composition using CONCOCT [47]. Assembled contigs were split in chunks of $10 \mathrm{~Kb}$ prior to read mapping using Bowtie2 [48]. Quality of the genome bins was assessed using CheckM [49] to determine genome completeness and contamination, which was based on the presence/absence of sets of colocalized single-copy genes present within a phylogenetic lineage. Bins presenting completeness greater than $75 \%$, contamination less than $10 \%$, and strain heterogeneity less than $25 \%$ were selected for further analysis using Phyla-AMPHORA [50], which also incorporates bacterial phylum-level phylogenetic markers for taxonomy assignment.

\section{Cultivation, isolation, and genomic-based characterization of microorganisms}

The LigMet culture broth was serially diluted and plated directly on agar media supplemented with 1:1 (v/v) LW lignin and $0.25 \%(\mathrm{w} / \mathrm{v})$ high-molecular-weight (HW) insoluble lignin, prepared as described by Rocha et al. [30]. After incubation of the plates at $30^{\circ} \mathrm{C}$ for $48 \mathrm{~h}$, 48 selected colonies were transferred again to agar medium supplemented with lignin fractions and incubated at the same condition. The colonies were then covered with a $1.5 \%(\mathrm{w} / \mathrm{v})$ agar solution supplemented with $0.01 \%(\mathrm{w} / \mathrm{v})$ Azure-B dye. Colonies showing lignolytic activity by the formation of dye decolorization halos around them were cultivated and their genomic DNA extracted as previously described. Identification was carried out based on the $16 \mathrm{~S}$ rRNA gene sequencing analysis using BLASTn search against the RDP database with $97 \%$ sequence identity and $E$-value of $1 \mathrm{e}-10$. For genome sequencing, the total DNA was sequenced by applying the paired-end and mate-pair library protocols on an Illumina MiSeq platform. Genome reads were first quality-filtered and assembled using Velvet [51], and SSPACE [52] for scaffolding using the matepairs reads. Pilon [53] was used to further improve the genome assembly. The assembled genome sequence was imported into the annotation platform Integrated Microbial Genomes (IMG/ER, [54]) for automatic prediction of genes. Finally, the assembled genome of the isolate was compared to the genome bins using Mauve [55] and Mummer (version 3.0 [56],) to determine their similarity.

\section{Data availability}

Raw sequencing reads of the amplicon, metagenome, and genome sequencing analysis have been deposited in the DDBJ/ENA/GenBank under the accession number PRJEB20169. The draft genome sequences of Paenarthrobacter sp. HW13 have been deposited in the IMG/ER under Study ID: Gs0118559. 


\section{Cloning and expression of genes involved in vanillin biosynthesis}

The biotransformation of ferulic acid into vanillin was based on expression of two genes, ferA_B3 (MG214406) and ferB_B11 (MG214407), encoding feruloyl coenzyme A synthetase and enoyl-CoA hydratase, respectively. These genes sequences were obtained from the LigMet metagenomic data set and synthesized by Biomatik (Biomatik Corporation, Canada). The ferB_B11 gene was inserted into the pET28a-vector, while the fer $A \_B 3$ gene was cloned in the pETTRXA-1a/LIC by the ligase independent cloning (LIC) method [57]. Cloning was verified by PCR and both constructions were transformed into Escherichia coli BL21(DE3) for protein expression. A His6-tag was fused at the N-terminal to promote purification in a His-Trap-Ni-NTA column (GE Healthcare) for both proteins. Details and specifications of the expression step, strains, and plasmids are described in detail in Additional file 1: Methods section).

\section{Enzymatic assays}

The enzymatic assays were performed according to methodology described in Yang et al. [58]. Briefly, the first reaction, conversion of ferulic acid into feruloylCoA, consisted of $100 \mathrm{mM} \mathrm{KH} \mathrm{PO}_{4}$ buffer ( $\mathrm{pH}$ 7.0), $2.5 \mathrm{mM} \mathrm{MgCl}_{2}, 1 \mathrm{mM}$ ferulic acid, $2 \mathrm{mM}$ ATP, $0.4 \mathrm{mM}$ coenzyme A, and $1.5 \mu \mathrm{g}$ of the purified protein FerA. The mixture was incubated at $30{ }^{\circ} \mathrm{C}$ for $1 \mathrm{~h}$. Next, the feruloyl-CoA was converted into vanillin by addition of $1.5 \mu \mathrm{g}$ of the purified protein FerB and incubated for $24 \mathrm{~h}$ at the same temperature. Samples were taken from last reaction for GC-MS (gas chromatography-mass spectrometry) quantitative analysis to detect the vanillin production. The substrates and cofactors were purchased from Sigma-Aldrich (St. Loius, MO, USA).

\section{Analytical methods for vanillin production}

To detect phenolics and vanillin production from enzymatic reactions, we used a quantitative GC-MS analysis. The resulting phenolics from enzymatic reactions were extracted by adjusting the $\mathrm{pH}$ of the samples to below 2 with $6 \mathrm{~mol} \mathrm{l}^{-1} \mathrm{HCl}$ and addition of butyl acetate (1:1, v:v) and then derivatized [59]. The derivatized samples $(1 \mu \mathrm{l})$ were analyzed on an Combi-PAL autosampler (Agilent Technologies GmbH, Waldbronn, Germany) coupled to an Agilent 7890 gas chromatograph in split less mode coupled to a Leco Pegasus two time-of-flight mass spectrometer (LECO, St. Joseph, MI, USA) as described by Weckwerth et al. [60]. The chromatograms were exported from the Leco ChromaTOF software (version 3.25) to the $\mathrm{R}$ software. Peak detection, retention time alignment, and library matching were performed using the Target Search R-package [61]. Metabolites were quantified by the peak intensity of a selective mass. The intensity normalization procedure was performed by dividing the fresh weight, followed by sum of the total ion count and global outlier replacement $[62,63]$.

\section{Results and discussion}

\section{Establishment of lignin-degrading consortium}

In Brazil, sugarcane straw is usually returned to the field after harvest. In the present study, the hypothesis under investigation was whether the sugarcane field soil covered by straws, after the plants were harvested, could be a potential source of novel lignolytic enzymes, because it is expected that the microbial population involved in degradation of plant biomass polymers is enriched in this environment (Fig. 1).

According to the previous studies, the establishment of microbial consortia is a powerful strategy to develop an enriched community with a particular metabolic ability, e.g., consortia for lignocellulosic biomass, phenanthrene, and bitumen degradation have been reported [21-23]. Thus, sugarcane field soil was used as inoculum in media containing minimal nutrients together with lignin fragments as the major carbon source. To establish a consortium able to degrade and modify lignin and/or lignin aromatic compounds, a sugarcane soil-derived consortium was adapted and enrichment on lignin medium for up to 50 consecutive weeks.

As illustrated in Fig. 2a, the liquid waste stream used for LigMet cultivation contains lignin fragments ranging from 300 to $1200 \mathrm{Da}$, including phenolic monomers such as ferulic and cinnamic acid (Additional file 1: Table S1), along with minimal sugar concentrations $\left(450 \mu \mathrm{M} \mathrm{l}^{-1}\right)$. According to Fig. 2b, it is possible to observe that compounds ranging from $\sim 700$ to $\sim 300 \mathrm{Da}$ decreased during the first $34 \mathrm{~h}$ of growth, indicating that the consortium was able to use the soluble lignin fragments as a carbon source. Moreover, during the first $40 \mathrm{~h}$, an increase in $\mathrm{OD}_{600}$ and consumption of sugars available in the medium was observed, indicating the growth of microbial community (Additional file 1: Figure S1).

\section{The LigMet structure evaluated through amplicon sequencing}

Alpha diversity is used to describe microbial community composition of a single sample. The calculated index, which includes species richness and diversity, allows to detect changes in its composition and to compare with other samples. Therefore, the microbial richness and diversity of the sugarcane soil and LigMet were quantified through amplicons sequencing. Statistical data summarizing the sequencing are shown in Additional file 1: Table S2. Clustering of $16 \mathrm{~S}$ rRNA gene amplicon resulted in 1558 and 355 of OTUs for the sugarcane soil and 

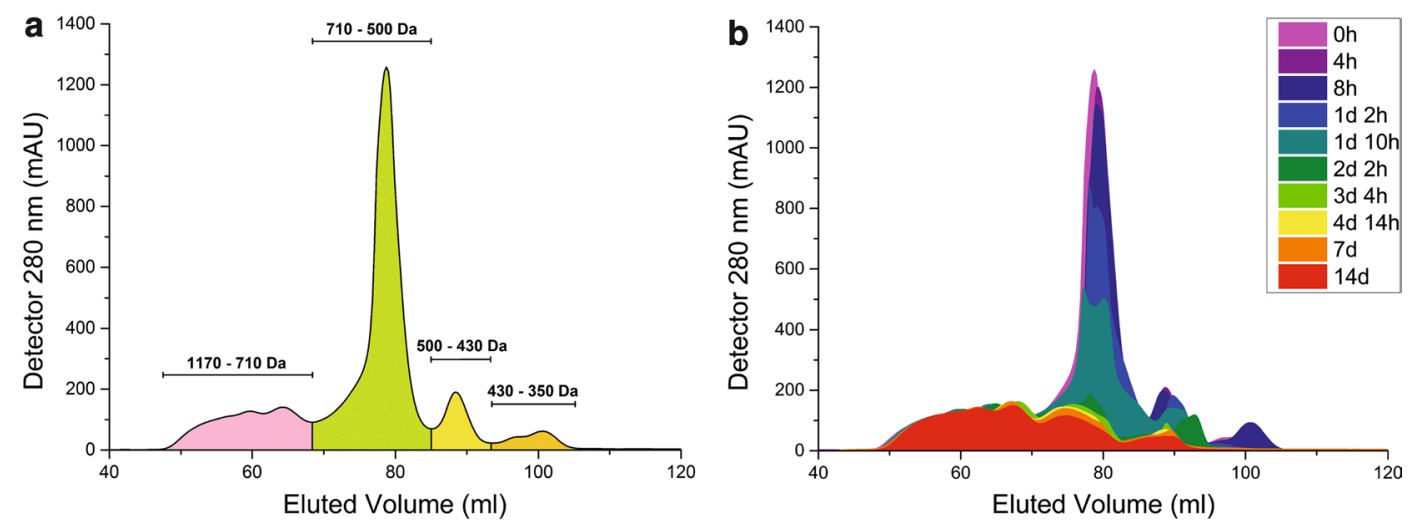

Fig. 2 GPC chromatograms showing the molecular weight distribution of a lignin-waste stream used as the sole carbon source for establishment of the lignin-degrading consortium (LigMet) and $\mathbf{b}$ high- and low-molecular-weight fragments detected during 14 days of LigMet cultivation. Above the peaks are the molecular weight distributions in Dalton (Da), which are inversely correlated with the elution time. Different phenolic compounds and polymers were used as internal standards

LigMet, respectively. ACE and Chao1 (richness estimators) and rarefaction analysis suggested that the bacterial species richness in soil and LigMet were entire covered (Additional file 1: Table S3 and Figure S2A, S2B, S3). Similarly, Good's coverage index of 0.99 for both LigMet and soil indicated that the sequencing was enough to cover the whole bacterial species. Moreover, Shannon and Simpson's diversity, which calculated species richness and evenness based on different taxa and their relative abundance, were higher for soil (6.3 and 0.99, respectively) in comparison to LigMet (3.4 and 0.92) (Additional file 1: Table S3). The lower microbial diversity, as well as richness and evenness, in enriched consortia is an expected selection response based on selective media [21, $22,24,27$ ] and the microorganisms best adapted become dominant [24]. In our study, four OTUs represented $48 \%$ of total sequences (Fig. 3b), consequently, decreasing the diversity in LigMet compared to its microbial source (soil).

To compare the taxonomic composition of LigMet and sugarcane soil, the OTU representative sequences were assigned from the phylum to genus level. Figure 3 shows the sugarcane soil and LigMet taxonomic profile. A total of 17 phyla were identified in soil amplicon data and the most abundant phyla were Proteobacteria (29\% of the total reads), Acidobacteria (22\%), and Actinobacteria (15\%) (Fig. 3a). At the class rank, Alphaproteobacteria (15\%), Actinobacteria (14\%), and Acidobacteria (14\%) were predominant in LigMet (Additional file 1: Figure S4). The overall taxonomic profile of the sugarcane soil corroborated with the previous sequencing studies addressing soil microbial communities [64-66].

The LigMet exhibited a significant difference in the relative abundance of the phylogenetic groups compared to the sugarcane soil. The LigMet $16 \mathrm{~S}$ sequences of Proteobacteria origin reached up to $58 \%$ of the total sequences classified, which were assigned to the Betaproteobacteria (25\%), Alphaproteobacteria (24\%), and Gammaproteobacteria (9\%) classes (Fig. 3a). Members of Actinobacteria and Firmicutes were also enriched in LigMet, representing the second and the third predominant phyla with $21 \%$ and $13 \%$ of the classified sequences, respectively. On the other hand, sequences assigned to Acidobacteria class decreased to less than $0.1 \%$ in the LigMet, while, in the soil, this group represented around $22 \%$ of the total sequences (Fig. 3a). The most abundant bacterial families included Alcaligenaceae (24\%), Micrococcaceae (11\%), Phyllobacteriaceae (9\%), and Paenibacillaceaea (5\%), totalizing $48 \%$ of the total sequences assigned. Interestingly, the predominant family in LigMet (Alcaligenaceae) was virtually non-existent in the soil (less than $0.5 \%$ ). At the genera level, almost half of total the sequences in the LigMet were assigned to Achromobacter (belongs to Betaproteobacteria), Paenarthrobacter (Actinobacteria), Pseudaminobacter (Alphaproteobacteria), and Paenibacillus (Bacilli). The result suggests that these microorganisms were key players in LigMet. Betaproteobacteria, Alphaproteobacteria, Actinobacteria, and Bacilli classes were frequently reported as degraders lignin and/or lignin-derived aromatic compounds [14, 17, 67, 68]. For example, Achromobacter and Paenarthrobacter have been characterized for their ability to degrade biphenyl and polycyclic aromatic hydrocarbons, respectively [69, 70]. Ochrobactrum, Rhizobiales, Sphingobium, and Sphingomonas are examples of the genus belong to Alphaproteobacteria described as lignin-degrading bacteria [14, 18, 69]. Nonetheless, 
a

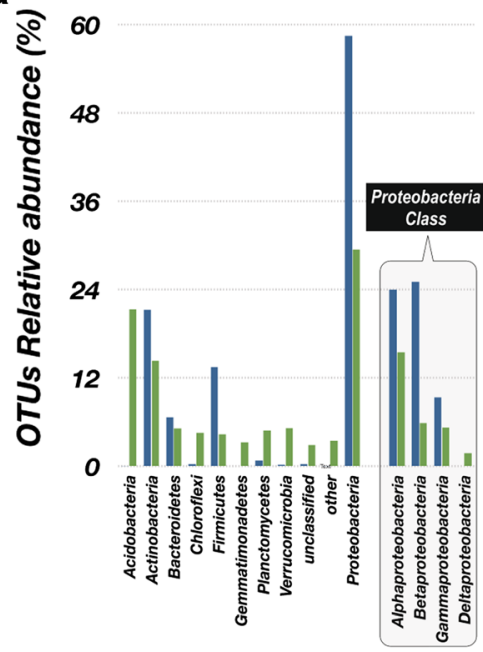

b

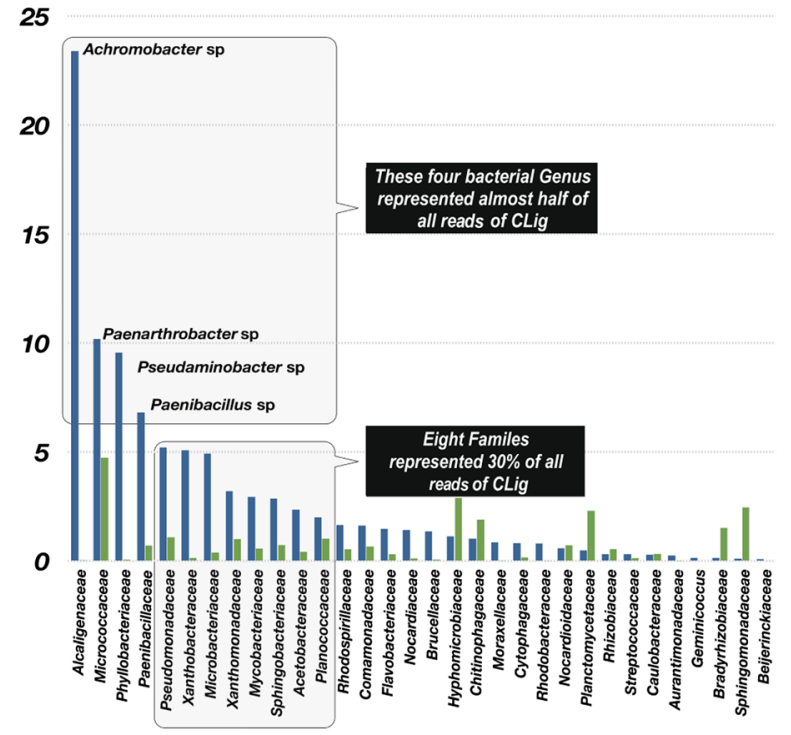

\section{LigMet Sugarcane soil}

C

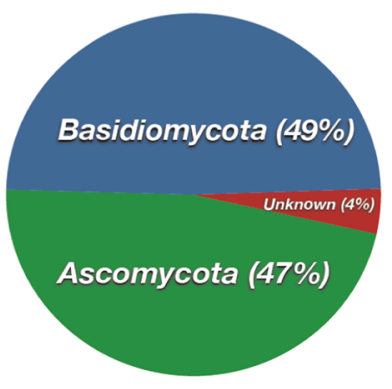

d

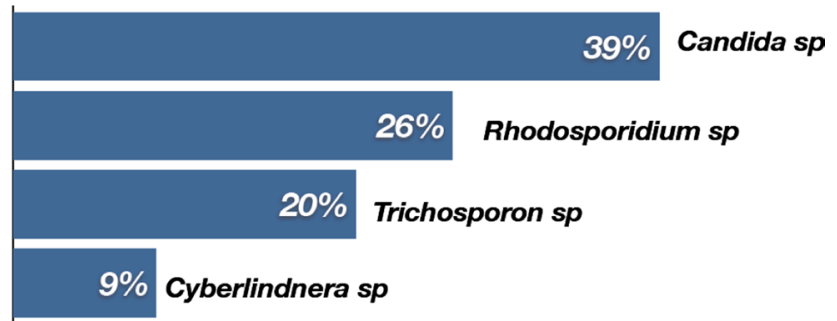

$6 \%$ unclassified

Fig. 3 Taxonomic composition of microbial communities from LigMet and the sugarcane soil samples. a Bacterial composition at phylum level. b Bacterial families of highest abundance in the LigMet and in the sugarcane soil sample. $\mathbf{c}$ and $\mathbf{d}$ Fungal communities at phylum and species level, respectively, from LigMet sample based on ITS2 sequencing

Pseudomaniobacter genus is being reported for the first time involved in aromatic compounds degradation.

The presence of fungal community was also analyzed in LigMet. The richness estimators indicated only 12 OTUs, considering an evolutionary distance of 0.03 , while Shannon and Simpson diversity were 1.44 and 0.71, respectively (Additional file 1: Table S3). OTUs were assigned to Basidiomycota and Ascomycota members (Fig. 3c). On average, approximately $94 \%$ of all the analyzed sequences could be assigned at the genus level (Fig. 3d). Among them, Candida sp. (39\%), followed by Rhodosporidium sp. (26\%), Trichosporon sp. (20\%), and Cyberlindnera sp. (9\%), were predominant. Candida tropicalis was described as being capable of degrading phenolic compounds [71, 72]. Trichosporon and Cyberlindnera were reported as dye-decolorizing yeasts [73].

The community had very few sequences assigned to the Archaea domain, such as OTUs assigned to the phylum
Thaumarchaeota, which was identified in sugarcane soil and LigMet with $0.038 \%$ and $0.002 \%$ of total relative OTU abundance, respectively (Additional file 1: Figure S5). The phylum Euryarchaeota was identified only in the LigMet (0.01\% relative abundance). Moreover, sequences affiliated with the protozoan class Litostomatea were detected in LigMet based on 18S rDNA analyses (Additional file 1: Table S4). According to Simek et al. [74] and Jürgens et al. [75], the taxonomic structure of microbial communities can be shaped by protozoa due to its preferential predation of particular bacterial taxa.

Overall, the statistical analysis showed the enrichment of a few phylogenetic groups in response to adaption of the carbon source added to the medium. As mentioned previously, the taxonomic analysis revealed several microorganisms previously reported as lignin and/or phenolic compounds degraders, thus encouraging further analyses on the genetic content of LigMet. 


\section{Genome assembly and overall functional annotation of LigMet}

A total of 97.5 million high-quality paired-end reads ( 18 gigabase of sequences) were generated from the LigMet sample. Assembled reads resulted in approximately 260 megabases (Mb) of contiguous sequences $\geq 1$ Kilobase (Kb). The N50 statistic from the assembled data was $35.5 \mathrm{~Kb}$ and the longest contig assembled reached up to $1.3 \mathrm{Mbp}$. Eukaryotic sequences were not detected in the assembled metagenome, and therefore, downstream analyses focused only on prokaryotic genes and genomes. Considering the complete assembly, 282,237 gene models were predicted (prokaryotic protein-coding regions), where 243,896 of these were larger than $300 \mathrm{bp}$.

The functional annotation was determined by different methods, where all predicted proteins obtained from the metagenome data set were annotated using EggNOG, KEGG, Pfam, and dbCAN databases. According to the results, 242,299 unigenes (85.8\% of the predicted proteins) were assigned based on the EggNOG database, 129,140 (45.7\%) based on KEGG, and 208,248 (73.78\%) on Pfam, and 8800 (3.1\%) had at least one protein domain annotated in dbCAN. The functional annotation obtained based on EggNOG and KEGG was quite similar (Fig. 4); the majority of proteins were classified as belonging to the metabolism category, which harbors a variety of pathways involved in degradation of aromatic compounds. According to the KEGG and Pfam annotation, several aromatic compound degradation pathways were found complete in the metagenome data set, including benzoate degradation (or methyl benzoate) to catechol (or methylcatechol), catechol ortho-cleavage, catechol meta-cleavage, and phthalate degradation (Additional file 1: Figure S6 and Fig. 5). The pathways for metabolism of lignin components and gene designations were described and demonstrated in the previous studies [13, 76-78].

\section{Reconstruction of draft genomes from the metagenomic data set}

The assembly of metagenomic data resulted in the reconstruction of 65 bins (Additional file 1: Table S5), of which 20 were considered draft bacterial genomes, since they presented completeness greater than $75 \%$ and contamination less than $10 \%$ (Table 1 ). The taxonomic assignments show that most of the genome bins were assigned to Alphaproteobacteria (9 bins), followed by Actinobacteria (6 bins) and Betaproteobacteria (2 bins). The remaining genome bins were assigned to Gammaproteobacteria, Bacilli, and Sphingobacteriia. These results are in accordance with the previous studies that reported Rhodococcus, Sphingobacterium, Ochrobactrum, Bacillus, Sphingobium, and Arthrobacter as microorganisms that

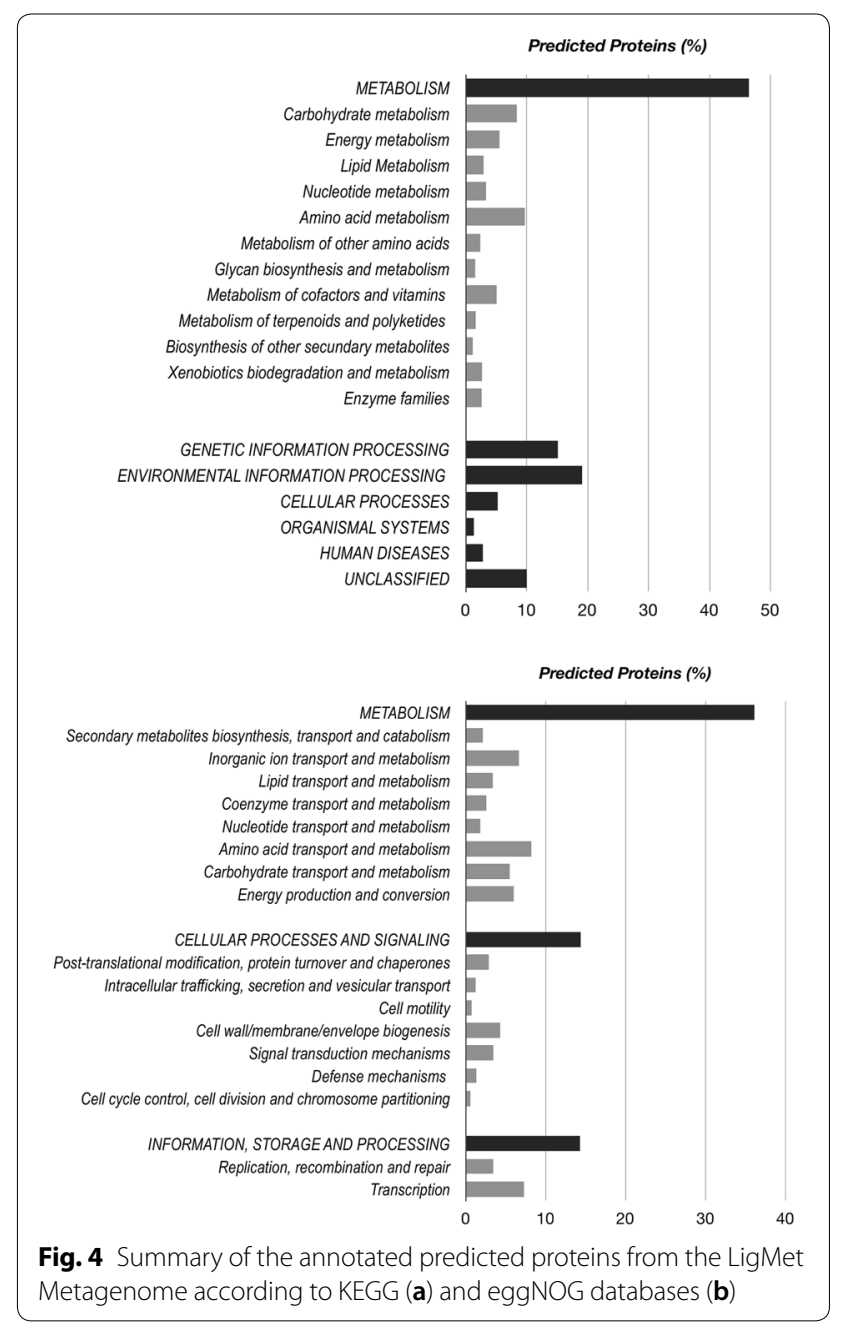

harbor lignolytic enzymes and metabolize aromatic compounds [14, 16, 79]. The $20 \mathrm{draft}$ genomes were further analyzed to depict their metabolic potential on lignin and aromatic compound degradation (Fig. 5).

\section{Biocatalysts and pathways involved in lignin degradation and aromatic compound conversion}

Pfam-based analysis identified several conserved domains related to lignin and aromatic compound degradation in the LigMet data set and genome bins (Fig. 5). Among these, peroxidases (PF00141), dye-decolorizing peroxidases (PF04261), and laccases (PF00394, PF02578, PF07731, and PF07732) domains, and enzymes that can generate radicals for cleavage of several lignin linkages, were found predominantly in genome bins belonging to the Actinobacteria class (bins 48,7,17, and 65) (Fig. 5). These findings corroborated the previous studies, reporting that Actinobacteria is able to produce extracellular lignolytic enzymes $[14,17,80]$. 


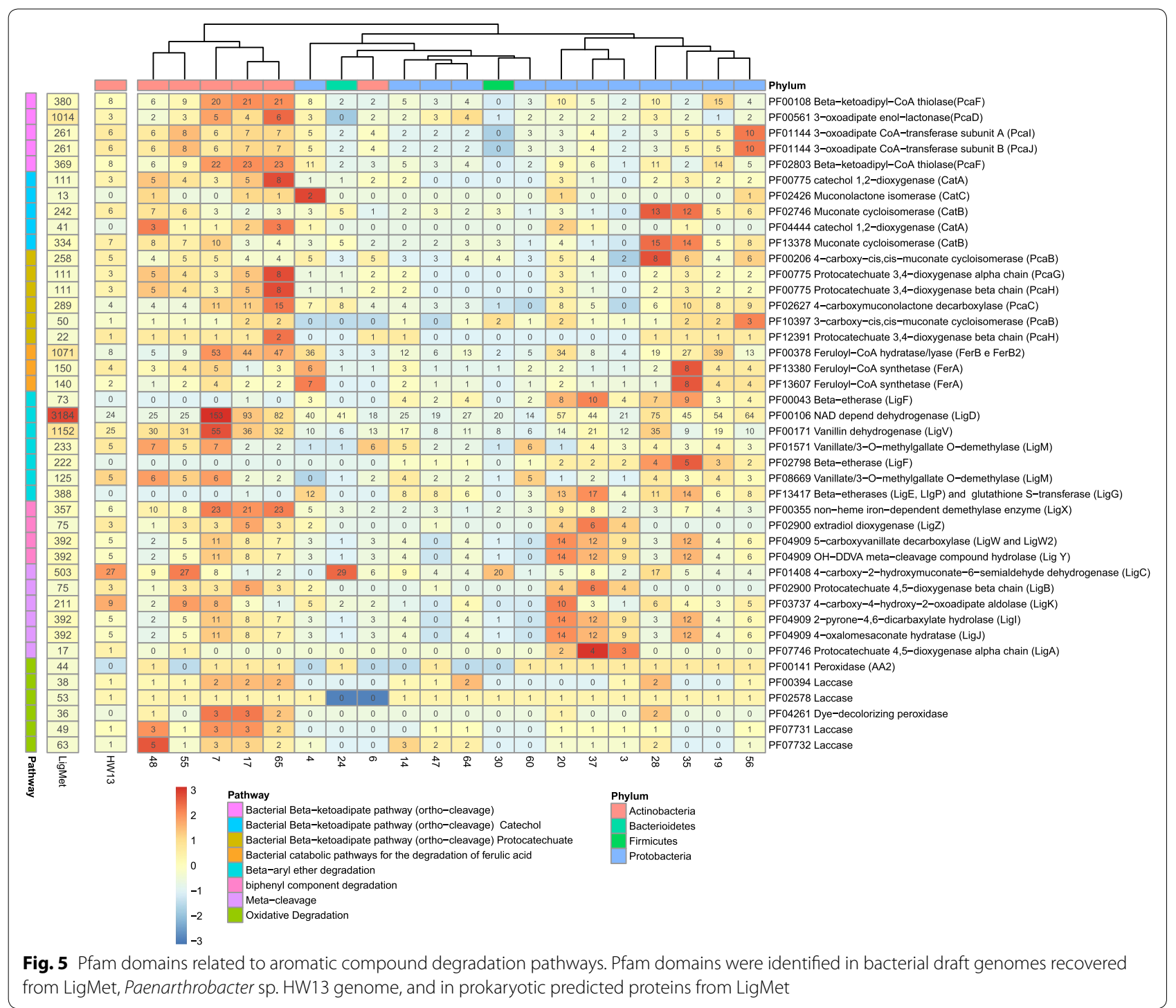

Glutathione-dependent beta-etherases of Sphingobium sp. were recently described as able to cleave beta-aryl ether bonds of lignin from softwoods and hardwoods [81]. Corresponding domains for the beta-etherases LigE, LigP, LigG, and LigF (considering the PF13417, PF00043, and PF02798) were predominantly found in bins 4, 20, 28, 35, and 37, which were assigned with Alpha and Betaproteobacteria origin (Fig. 5). Conversion of the beta-aryl ether lignin dimer involves LigEFG and LigD (PF00106) and results in vanillic acid [16], which can also be generated from vanillin by the action of LigV (PF00171). In this pathway, vanillic acid is converted into protocatechuic acid by LigM (PF01571 and PF08669). All these protein domains (LigEFG, LigD, LigV, and LigM) were identified in several bins, indicating their potential to metabolize vanillin and vanillic acid. Nonetheless, they were found predominantly in bins assigned to Mycobacterium (bin 7).
The biphenyl linkage is also a common component of lignin structure. Its cleavage is carried out by the cascade pathway LigX (PF0035), LigZ (PF02900), and LigW, LigW2, and LigY (PF04909) [16], also resulting in the central intermediate vanillic acid. With the exception of bins 6 and 28, several Actinobacteria genome bins displayed the complete set of proteins needed for biphenyl and vanillic acid conversion (Fig. 5). However, it is possible to notice that this cleavage cascade pathway was identified in only a few bins of Proteobacteria origin (20, 37, and 3) (Fig. 5).

The depolymerization of lignin releases a mixture of aromatic monomers that can be used as carbon and energy sources by several microorganisms [82, 83]. Phenolic aromatic monomers can be converted into metabolic intermediates, via catechol and protocatechuate, by the action of dioxygenases, which are classified according 
Table 1 Assembly statistics from draft genomes binned from CLig metagenome (20 best results)

\begin{tabular}{|c|c|c|c|c|c|c|c|c|}
\hline Bin Id & Taxonomic affiliation & $\begin{array}{l}\text { Completeness } \\
\text { (\%) }\end{array}$ & $\begin{array}{l}\text { Contamination } \\
\text { (\%) }\end{array}$ & $\begin{array}{l}\text { Heterogeneity } \\
\text { (\%) }\end{array}$ & Total length (bp) & \# contigs & $\begin{array}{l}\# \\
\text { Predicted } \\
\text { genes }\end{array}$ & GC (\%) \\
\hline 3 & Sphingomonadaceae & 97.9 & 2.1 & 0 & $2,600,224$ & 14 & 2487 & 63.8 \\
\hline 4 & Alcaligenaceae & 96.6 & 3.2 & 8.3 & $4,071,016$ & 139 & 3831 & 63.9 \\
\hline 6 & Microbacteriaceae & 93.0 & 2.3 & 25.0 & $2,775,244$ & 23 & 2744 & 70.2 \\
\hline 7 & Mycobacterium sp. & 98.6 & 4.7 & 7.4 & $8,113,983$ & 152 & 7824 & 67.0 \\
\hline 14 & Agrobacterium sp. & 97.0 & 1.9 & 0 & $3,723,529$ & 25 & 3637 & 62.4 \\
\hline 17 & Rhodococcus sp. & 96.4 & 1.4 & 12.5 & $5,940,093$ & 80 & 5417 & 67.9 \\
\hline 19 & Rhodobacter sp. & 97.5 & 3.5 & 0 & $4,829,944$ & 102 & 4636 & 66.4 \\
\hline 20 & Comamonadaceae & 76.1 & 1.8 & 0 & $5,317,169$ & 797 & 5668 & 69.5 \\
\hline 24 & Sphingobacterium sp. & 92.2 & 2.2 & 0 & $5,342,826$ & 905 & 5155 & 40.9 \\
\hline 28 & Ochrobactrum sp. & 99.1 & 10.5 & 0 & $8,098,959$ & 173 & 7885 & 56.5 \\
\hline 30 & Bacillus sp. & 92.6 & 3.7 & 0 & $4,962,376$ & 118 & 4977 & 35.4 \\
\hline 35 & Acetobacteraceae & 96.7 & 2.0 & 0 & $5,217,664$ & 48 & 5050 & 70.2 \\
\hline 37 & Sphingobium sp. & 98.1 & 9.3 & 5.7 & $6,313,527$ & 428 & 6265 & 59.7 \\
\hline 47 & Pseudoxanthomonas sp. & 97.3 & 1.5 & 0 & $3,192,941$ & 22 & 2886 & 71.6 \\
\hline 48 & Paenarthrobacter sp. & 97.0 & 4.1 & 0 & $4,259,515$ & 57 & 3930 & 68.2 \\
\hline 55 & Paenarthrobacter sp. & 97.3 & 1.6 & 0 & $4,537,449$ & 80 & 4185 & 63.5 \\
\hline 56 & Acetobacteraceae & 92.7 & 1.7 & 25.0 & $5,069,773$ & 252 & 4671 & 70.4 \\
\hline 60 & Hyphomicrobium sp. & 97.9 & 0.9 & 0 & $3,563,628$ & 12 & 3278 & 64.7 \\
\hline 64 & Methylobacterium sp. & 94.8 & 1.6 & 25.0 & $3,854,043$ & 176 & 3682 & 70.1 \\
\hline 65 & Rhodococcus & 96.8 & 1.8 & 0 & $5,770,250$ & 74 & 5292 & 70.8 \\
\hline
\end{tabular}

to the relative positions of hydroxyl groups (ortho- and meta-cleavage) [13]. The routes can be divided in three blocks: (i) the branch of catechol intermediate (orthocleavage), which involves the following enzymes: CatA (PF0775 and PF04444), CatB (PF02746 and PF13378), and CatC (PF02426); (ii) the branch of protocatechuate (metacleavage), which involves PcaG (PF00775), PcaH (PF00775 and PF12391), PcaB (PF10397 and PF00206), and PcaC (PF02627); and finally, (iii) the reactions common for both branches, catalyzed by PcaD (PF00561), PcaI (PF01144), PcaJ (PF01144), and PcaF (PF02803 and PF00108). Three Actinomycetes genome bins (17, 48, and 65) and two of Proteobacteria origin (4 and 20) presented all protein domains related to routes $\mathrm{i}$, ii, and iii. Regarding route (ii), all Actinobacteria genome bins and two of Proteobacteria origin (bins 20 and 35) were clearly enriched with coding genes involved in protocatechuate degradation. With exception of the Sphingobacterium genome bin (24) and the Bacillus bin (30), the protein domains corresponding to enzymes related to route iii were mapped in all bins.

The present work disclosed several novel aromaticdegrading enzymes of a high and low degree of homology to previously identified variants. For instance, the BLASTp comparison with beta-etherase (LigE) from $S$. paucimobilis SYK-6 identified 18 orthologous in LigMet, with sequence identity varying from 31 to $64 \%$ (E-value from $4 \mathrm{e}-30$ to 0.0$)$. Accordingly, LigMet orthologous to beta-etherase LigF (26 hits), Glutathione S-transferase homolog LigG (3 hits) and beta-etherase LigP (18 hits) from S. paucimobilis were recovered as well, with sequence identity varying from 26 to $93 \%$ ( $4 \mathrm{e}-178$ to $9 \mathrm{e}-11), 29$ to $84 \%(4 \mathrm{e}-164$ to $3 \mathrm{e}-18)$, and 31 to $88 \%$ $(4 \mathrm{e}-30$ to 0.0$)$, respectively.

Moreover, to obtain deeper insight on potential of the microbial community to carry out redox and hydrolytic mechanisms related to lignocellulose degradation, the auxiliary activity (AA) and carbohydrate esterase (CE) families, which act with CAZymes, were predicted based on dbCAN analysis (Additional file 1: Figure S7). The carbohydrate esterases from family 1 (CE1), including feruloyl esterases and $p$-coumaroyl esterases, can break down ester cross links of lignin and hemicelluloses [84, 85]; and glucuronyl esterases (CE15) were reported to catalyze ester linkage hydrolysis between glucuronoxylan and lignin [86]. Furthermore, acetyl esterases (described in families CE01, 02, 03, 04, 05, 06, 07, 12, and 16) play a role in degradation of xylose units esterified with acetic acid [85]. Nonetheless, only the families CE1 and CE4 were predominant in the LigMet metagenome data set and widespread in all genome bins (Additional file 1: Figure S7 and S8). Similar to CE families, AA were abundant in the LigMet metagenome, notably the AA3 family members, which may be related to peroxidase activity, thus corroborating with the previous PFAM-based functional analysis. 


\section{Paenarthrobacter sp. HW13 genome}

To complement the metagenomic discovery approach, the isolation of a novel lignin-degrading microorganism from LigMet was performed using HW or LW as the sole carbon source in media. Among the obtained colonies, only a single strain cultivated on HW exhibited a decolorization halo after being covered with an agar solution supplemented with Azure-B dye. Based on the 16S rRNA gene comparison, the analysis revealed that the strain HW13 shares 96.8\% 16S rRNA gene sequence similarity with $P$. ureafaciens DSM $20126^{\mathrm{T}}$ as the closest related species with a valid nomenclature (Additional file 1: Figure S9). Therefore, the strain may represent a new species within the Paenarthrobacter genus. The strain was denominated Paenarthrobacter sp. HW13.

The genome sequence analysis of the strain HW13 depicted its potential to metabolize lignin fragments and phenolic compounds (Fig. 6). Two libraries were sequenced on an Illumina/MiSeq system, resulting in 3,746,638 pair-end reads and 1,671,986 mate-pair reads, accounting for $1083 \mathrm{Mb}$. After data processing, the assembly resulted in three contigs consisting of 4,091,031 bases and featuring a CG content of $63 \%$. Additional details of the genome features can be found in Additional file 1: Table S6. To determine whether strain HW13 correspond to bin 48 or 55 (both assigned to the Paenarthrobacter genera), the microbial species identified (MiSi) method [87] was conducted, as implemented in IMG/ ER. The P probability of bins 48 and 55 being assigned to the same species as HW13 was 0.0 and 0.99 , respectively. Moreover, strain HW13 showed 100\% 16S rRNA gene sequence identity to the $16 \mathrm{~S}$ rRNA gene found in genome bin 55. The genomic analysis confirmed the prediction of several previously identified genetic determinants involved in aromatic compound metabolism, including the protocatechuate, catechol, phenylacetate, gentisate, and phenylpropionate degradation pathways (Fig. 6).

\section{Biosynthesis of vanillin from ferulic acid}

The occurrence of aromatic conversion pathways in LigMet to produce high value chemicals was validated through biochemical vanillin production. Ferulic acid is found in lignocellulosic biomass, and it is a precursor for biovanillin production [88]. The conversion of ferulic acid into vanillin has been reported in several microorganisms via coenzyme A-dependent, a non-beta-oxidative pathway [58, 89-91], including feruloyl-CoA synthetase (FerB) and enoyl-CoA hydratase/aldolase, (FerB) [92], which are regulated by $f e r C$, an MarR-type transcriptional regulator [93]. In this study, the protein domains related to FerA (PF13380) and FerB (PF00378) were found in several bins described in Fig. 5. The clusters of genes related to ferulic acid conversion into vanillin were manually identified in the LigMet, two presenting similar genetic structures previously described in P. fluorescens BF13 [93] and S. paucimobilis SYK-6 [78] (Fig. 7). In addition, another gene cluster was found in LigMet of similar organization to the gluconate operon of B. subtilis [94], which is regulated by a GntR family protein (Fig. 7).

Candidates' genes for fer $A$ (derived from bin 3 ) and $\operatorname{fer} B$ (derived from bin 11) were selected for further analyses. The FerA_B3 (bin 3) when compared to other bacterial feruloyl-CoA synthetases displays 60\% amino acid identity to S. paucimobilis (A0A031JK39), followed by Sphingobium sp. (60\%; BAK67177), Delftia acidovorans (55\%; CAC83622), and Burkholderia glumae (52\%; ACR31088). The FerB_B11 (bin 11) compared to other bacterial enoyl-CoA hydratases shows $63 \%$ amino acid identity to Pseudomonas nitroreducens (C3VA24), followed by Amycolatopsis methanolica (59\%; A0A076MUC3), Streptomyces sp. (55\%; S5LPF1), and D. acidovorans (55\%; Q8VNW7). Amino acid sequences from NCBI database and Uniprot with similarity higher than 20\% to FerA_B3 and FerB_B11 were considered to construct phylogenetic trees (Additional file 1: Figure S10). It evidenced the relationship FerA_B3 and FerB_B11 with other bacterial feruloyl-CoA synthetases and enoyl-CoA hydratases, respectively, but the low bootstrap value at most of the clades denoted the high divergence of FerA_B3 and FerB_B11.

For the bioproduction of vanillin, candidates genes for fer $A$ and $f e r B$ were then synthesized and successfully cloned for expression in $E$. coli, followed by purification by liquid chromatography. The conversion of ferulic acid into vanillin, after combining the two purified enzymes, was confirmed by GC-MS. The vanillin production from ferulic acid was detected after 6 and $24 \mathrm{~h}$ incubation using the purified recombinant proteins (Fig. 8). The biochemical and structural characterization of the two proteins, FerA_B3 and FerB_B11, will be the focus for future studies.

\section{Conclusions}

Different approaches were applied to validate the establishment of a lignin-degrading consortium and applicability of the metagenomic strategy. From the analysis of massive DNA sequencing data, several microorganisms and enzymes linked to lignin-degrading metabolic pathways were identified in LigMet. Furthermore, gene clusters involved in the non-beta-oxidative pathway for vanillin production were depicted, followed by production of recombinant FerA and FerB in functional form for successful production of the phenolic aldehyde. The genome sequencing analysis of a bacterium revealed eight gene clusters encoding proteins related to aromatic degradation, suggesting potential for biotechnological application. 


\section{Phenylacetate or phenylpropionate}

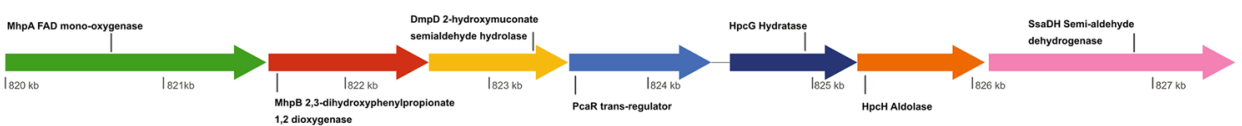

Gentisate/salicylic acid

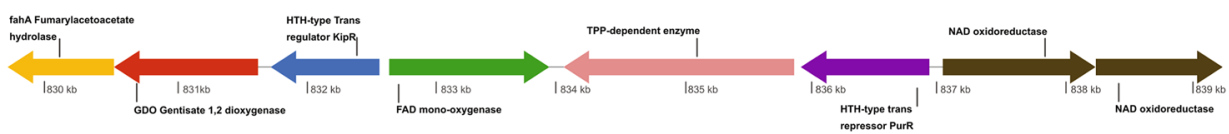

Meta-cleavage

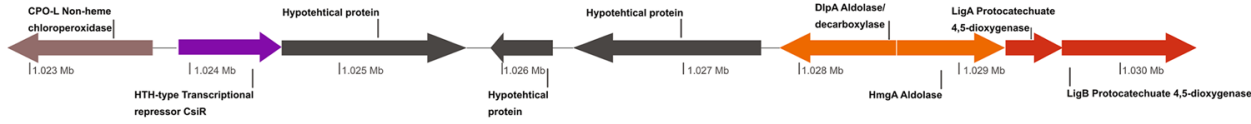

Phthalate

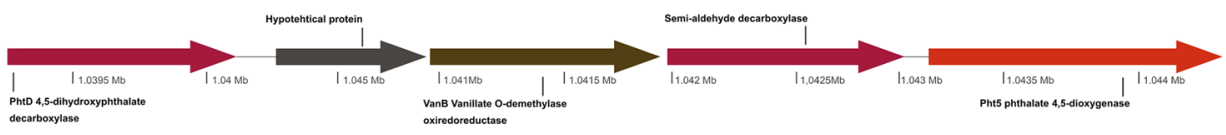

Beta-ketoadipate

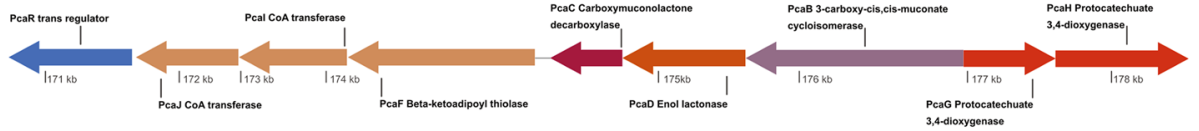

Possible biphenyl

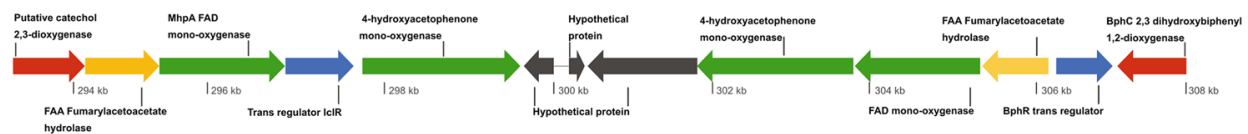

Phenylpropionate

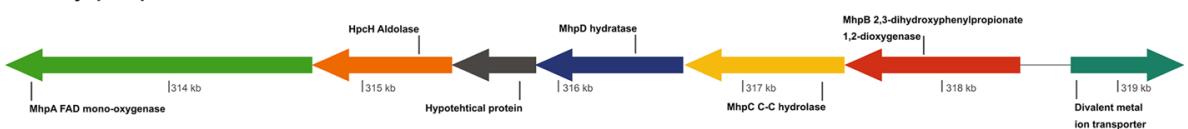

Phenylacetate
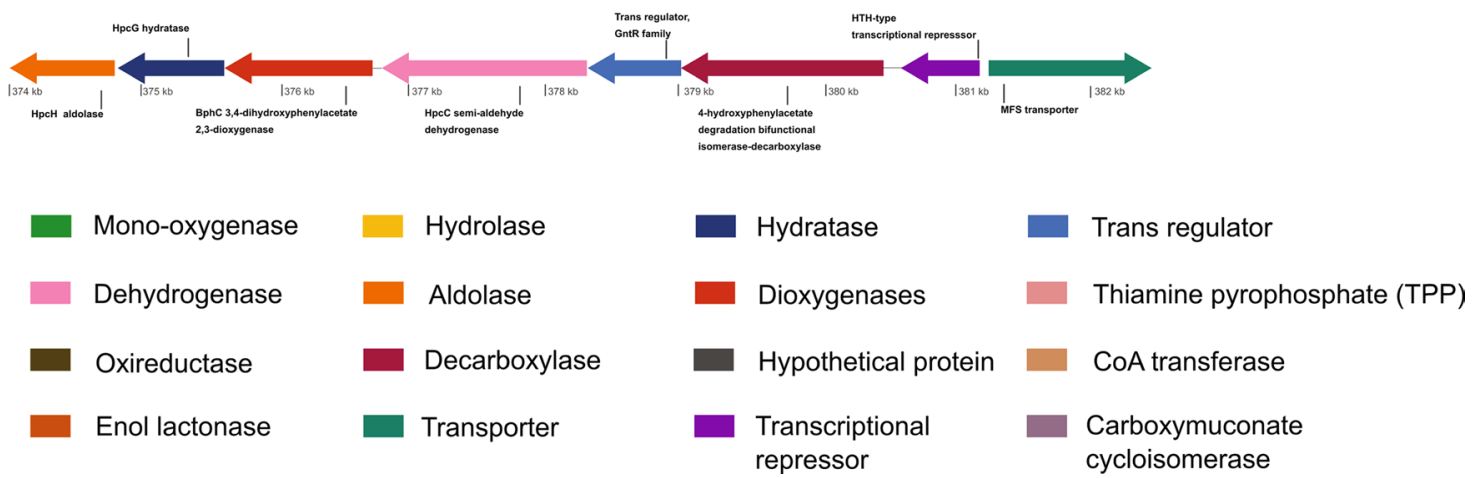

Fig. 6 Schematic representation of gene clusters involved in aromatic degradation pathways identified in the Paenarthrobacter sp. HW13 genome. Homologous genes are shown in the same color and the predicted gene products are indicated 


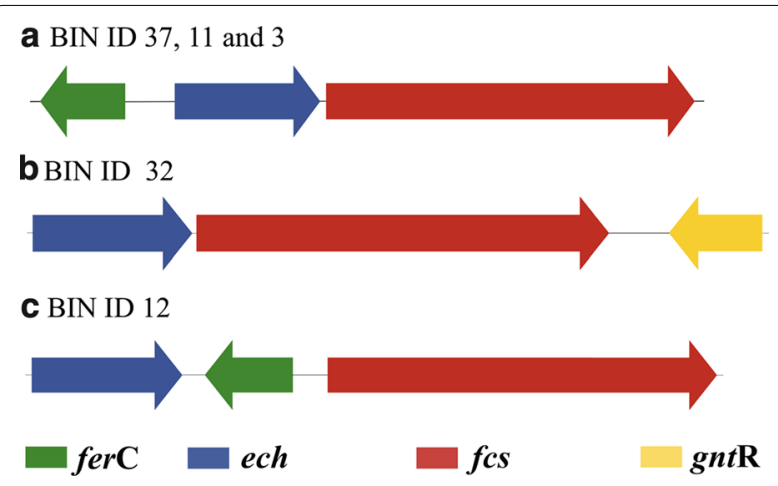

Fig. 7 Structure of the FerA and FerB genes clusters identified in the LigMet metagenome. The structure of FerA and FerB genes clusters described in a Sphingobium sp. and $\mathbf{b}$ Bacillus subtilis, in which the regulator gene belongs to the GntR family, and c Pseudomonas sp., showing different arrangements of the regulator (FerC). The three gene cluster configurations were identified in the LigMet [17-19], as well as on draft genomes recovered from LigMet, as indicated. Homologous genes are shown in the same color and the predicted gene products are indicated

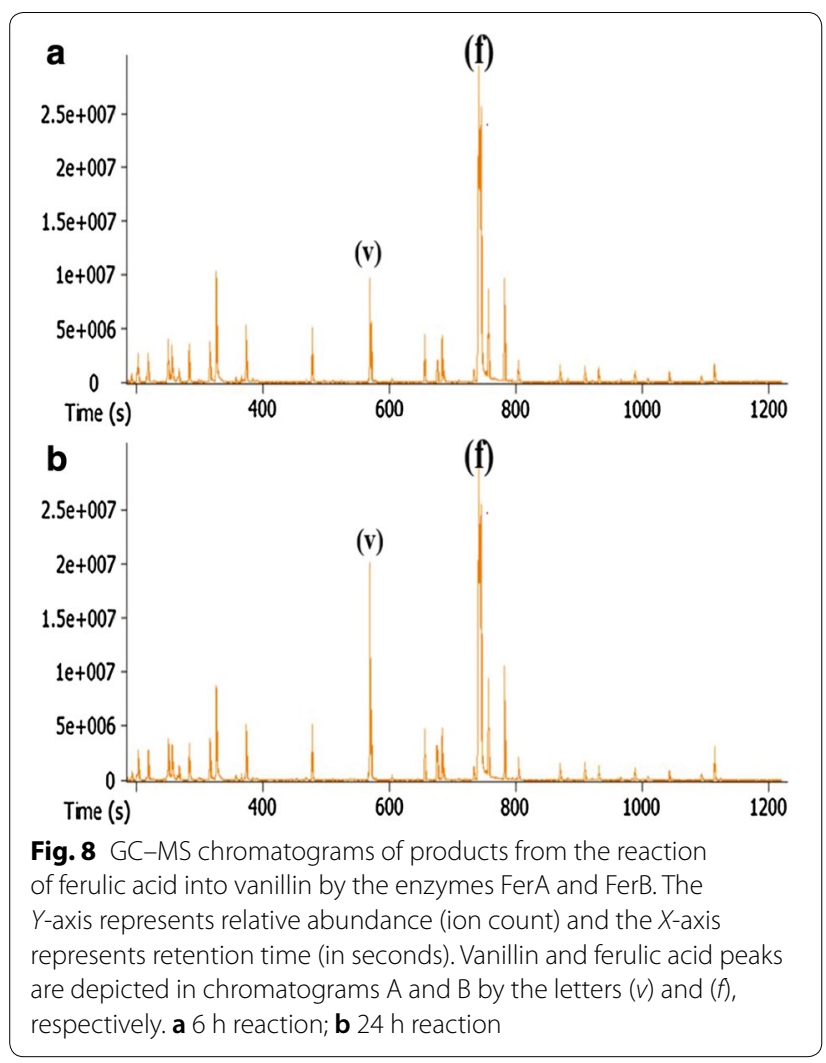

The LigMet metagenome represents a vast reservoir of genes coding for enzymes involved in lignin depolymerization and assimilation. The targeted metagenomic discovery platform described in the present study is of potential interest to reveal optimized gene sets and microorganisms for initiatives based on synthetic biology principles to produce high value chemicals from lignocellulose. The straightforward metagenomic strategy described could also be applied to other fields such as the development of antibiotic producing microorganisms or the recycling of plastic polymers.

\section{Additional file}

Additional file 1: Table S1. Compounds identified by gas chromatography-mass spectrometry (GC-MS) in the lignin-waste stream used for establishment of the lignin-degrading microbial community (LigMet). Table S2. Sequencing statistics and data processing of amplicon libraries constructed for profiling LigMet and soil samples analyzed. Table S3. Diversity and richness indices of the LigMet and soil samples based on $16 S$ rRNA and ITS2 region sequences. Table S4. Protozoa identified in LigMet based on 18S rRNA sequencing. Table S5. Assembly statistics from draft genomes recovered from LigMet (all assemblies). Table S6. Genome statistics of Paenarthrobacter sp. str. HW13. Figure S1. Microbial growth was monitored by OD $600 \mathrm{~nm}$, observing exponential growing during the first 40 hours of consortium growth. The consumption of reducing sugars over time as monitored by DNS, the exponential phase was completed after the first 40 hours of growth when monitoring sugar consumption. Figure S2. Rarefaction curves based on targeted sequencing of 165 rRNA gene amplicons derived from the LigMet $(\mathbf{A})$ and sugarcane soil (B) samples. The rarefaction curves of each biological replicate are shown in different colors. Figure S3. Rarefaction curves based on targeted sequencing of the ITS2 region derived from the LigMet sample. The rarefaction curves of each biological replicate are shown in different colors. Figure S4. The taxonomic profiles from LigMet and sugarcane soil samples at the class level based on $16 \mathrm{~S}$ rRNA gene amplicon. The respective relative abundances of each biological replicate for LigMet and sugarcane soil are shown. Figure S5. The archaeal phylum abundance in LigMet and sugarcane soil sample. The relative abundance is shown in percentage for each biological replicate of the LigMet and sugacarcane soil. Figure S6. Metabolic pathways related to aromatic compound degradation identified in LigMet according to KEGG automatic annotation. Figure S7. Classification of the predicted proteins from the LigMet according to the $\mathrm{dbCAN}$ database. Figure S8. Predicted auxiliary activity (AA) and carbohydrate esterase (CE) families from LigMet and draft genomes, based on the $\mathrm{dbCAN}$ database. AA and CE families are related to peroxidase activity and break down of lignin ester cross links, respectively. Figure S9. Phylogenetic relationship of the strain HW13 relative to the most closely related strains of the genus Paenarthrobacter. EzBioCloud webserver was used to perform a similarity-based search of HW13 16S rRNA to retrieve the most closely related sequences. The resulting $16 \mathrm{~S}$ rRNA sequences were aligned using the MAFFT v7.299b software. A phylogenetic tree was inferred using the maximum likelihood method implemented in RAxML v8.2.0, evolutionary distances were based on the GTRGAMMAI model, inferred as the best model by jModelTest2. Numbers at the nodes are percentages of bootstrap values obtained by repeating the analysis 1,000 times. The type strains are marked with a superscript ' $T$ '. Accession numbers are shown in parentheses. Figure S10. Phylogenetic relationships among feruloyl-CoA synthetase (upper) and Enoyl-CoA hydratase/aldolase. The phylogenetic tree was generated using amino acid sequences retrieved from NCBI and Uniprot database. The sequences were aligned using MAFFT v7.299b software [5]. The phylogenetic tree was reconstructed using maximum likelihood method implemented in RAxML v8.2.0 [6], evolutionary distances were based on the GTRGAMMAI model, inferred as the best model by jModelTest2 [7]. The bootstrap values (1,000 replicate runs, shown as $\%)$ higher than $70 \%$ are represented. Accession numbers are listed in parentheses. The FerA_B3 and FerB_B11 amino acid sequence retrieved from LigMet data set is printed in bold. 


\section{Abbreviations}

LigMet: lignin-degrading consortium; DNS: 3,5-dinitrosalicylic acid; PCR: polymerase chain reaction; 165 rDNA: 165 rRNA gene sequence; ITS: nuclear ribosomal internal transcribed spacer; OTU: operational taxonomic unit; BLAST: Basic Local Alignment Search Tool; RDP database: ribosomal database project database; Pfam: protein families; dbCAN: database for carbohydrateactive enzyme annotation; KEGG: Kyoto encyclopedia of genes and genomes; eggNOG: evolutionary genealogy of genes: Non-supervised Orthologous Groups; MiSi: microbial species identified; HW: high molecular weight; CAZyme: carbohydrate-active enzyme; GH: glycoside hydrolase; AA: auxiliary activities; CE: carbohydrate esterase; HMM: hidden Markov model; GC-MS: gas chromatography-mass spectrometry.

\section{Authors' contributions}

ECM, TMA, GFP, DAAP, LBB, JAA, and GCE performed the experiments. TDHB, $C C$, and ND contributed with reagents, materials, and analyses. EMM, TMA, GFP, GT, and FMS analyzed the results and wrote the manuscript. ND and FMS revised the manuscript. All authors agreed with the submitted version of the paper. All authors read and approved the final manuscript.

\section{Author details}

${ }^{1}$ Laboratório Nacional de Ciência e Tecnologia do Bioetanol, Centro Nacional de Pesquisa em Energia e Materiais, Campinas, Brazil. ${ }^{2}$ Master Program in Industrial Biotechnology, Universidade Positivo (UP), Curitiba, Brazil. ${ }^{3}$ Manchester Institute of Biotechnology, School of Chemistry, University of Manchester, Manchester, UK. ${ }^{4}$ Department of Chemistry, University of Warwick, Coventry, UK. ${ }^{5}$ Programa de Processos Tecnológicos e Ambientais, Universidade de Sorocaba, Sorocaba, Brazil

\section{Acknowledgements}

E.C.M., G.T., and LBB were supported by grants from the São Paulo Research Foundation (FAPESP, proc. numbers 2014/26152-4, 2015/23279-6, and 2013/03061-0). ND is supported by the BBSRC David Phillips Fellowship (BB/ K014773/1) and BBSRC-FAPESP (FAPPA) grant (BB/L026244/1). ND and TDHB are both supported by BBSRC-FAPESP grant BB/P01738X/1. FMS is supported by the FAPESP grant (2015/50590-4). We would like to thank Brazilian Bioethanol Science and Technology Laboratory CTBE NGS Sequencing Facility for generating the sequencing data described here.

\section{Competing interests}

The authors declare that they have no competing interests.

\section{Ethics approval and consent to participate}

Not applicable.

\section{Publisher's Note}

Springer Nature remains neutral with regard to jurisdictional claims in published maps and institutional affiliations.

\section{Received: 23 October 2017 Accepted: 9 March 2018}

Published online: 22 March 2018

\section{References}

1. Alonso DM, Bond JQ, Dumesic JA. Catalytic conversion of biomass to biofuels. Green Chem. 2010;12:1493-513.

2. Caspeta L, Buijs NAA, Nielsen J. The role of biofuels in the future energy supply. Energy Environ Sci. 2013;6:1077.

3. Cheng S, Zhu S. Lignocellulosic feedstock biorefinery - the future of the chemical and energy industry. BioResources [Internet]. 2009;4(2):456-7.

4. Boerjan W, Ralph J, Baucher M. Lignin biosynthesis. Annu Rev Plant Biol. 2003;54:519-46.

5. Bruijnincx PCA, Weckhuysen BM. Biomass conversion: lignin up for breakdown. Nat Chem Nat Publ Group. 2014;6:1035-6.

6. Ragauskas AJ, Beckham GT, Biddy MJ, Chandra R, Chen F, Davis MF, et al. Lignin valorization: improving lignin processing in the biorefinery. Science. 2014:344:1246843.
7. Beckham GT, Johnson CW, Karp EM, Salvachúa D, Vardon DR. Opportunities and challenges in biological lignin valorization. Curr Opin Biotechnol. 2016:42:40-53.

8. Brenelli LB, Mandelli F, Mercadante AZ, de Rocha GJM, Rocco SA, Craievich AF, et al. Acidification treatment of lignin from sugarcane bagasse results in fractions of reduced polydispersity and high free-radical scavenging capacity. Ind Crops Prod. 2016;83:94-103.

9. Rahimi A, Azarpira A, Kim H, Ralph J, Stahl SS. Chemoselective metal-free aerobic alcohol oxidation in lignin. J Am Chem Soc. 2013;135:6415-8.

10. Rahimi A, Ulbrich A, Coon JJ, Stahl SS. Formic-acid-induced depolymerization of oxidized lignin to aromatics. Nature. 2014;515:249-52.

11. Gall DL, Ralph J, Donohue TJ, Noguera DR. Biochemical transformation of lignin for deriving valued commodities from lignocellulose. Curr Opin Biotechnol. 2017:45:120-6.

12. Christopher LP, Yao B, Ji Y. Lignin biodegradation with laccase-mediator systems. Front Energy Res. 2014;2:1-13.

13. Bugg TDH, Ahmad M, Hardiman EM, Rahmanpour R. Pathways for degradation of lignin in bacteria and fungi. Nat Prod Rep. 2011;28:1883-96.

14. Taylor CR, Hardiman EM, Ahmad M, Sainsbury PD, Norris PR, Bugg TDH. Isolation of bacterial strains able to metabolize lignin from screening of environmental samples. J Appl Microbiol. 2012;113:521-30.

15. Dashtban M, Schraft H, Syed TA, Qin W. Fungal biodegradation and enzymatic modification of lignin. Int J Biochem Mol Biol. 2010;1:36-50.

16. Bugg TDH, Ahmad M, Hardiman EM, Singh R. The emerging role for bacteria in lignin degradation and bio-product formation. Curr Opin Biotechnol. 2011;22:394-400.

17. Wang L, Nie Y, Tang Y-Q, Song X-M, Cao K, Sun L-Z, et al. Diverse bacteria with lignin degrading potentials isolated from two ranks of coal. Front Microbiol. 2016;7:1-14.

18. Masai E, Katayama Y, Nishikawa S, Fukuda M. Characterization of Sphingomonas paucimobilis SYK-6 genes involved in degradation of lignin-related compounds. J Ind Microbiol Biotechnol. 1999;23:364-73.

19. Zhou Y, Pope PB, Li S, Wen B, Tan F, Cheng S, et al. Omics-based interpretation of synergism in a soil-derived cellulose-degrading microbial community. Sci Rep. 2014;4:1-6.

20. Scully ED, Geib SM, Hoover K, Tien M, Tringe SG, Barry KW, et al. Metagenomic profiling reveals lignocellulose degrading system in a microbial community associated with a wood-feeding beetle. PLoS ONE. 2013;8:1-22.

21. Zhu N, Yang J, Ji L, Liu J, Yang Y, Yuan H. Metagenomic and metaproteomic analyses of a corn stover-adapted microbial consortium EMSD5 reveal its taxonomic and enzymatic basis for degrading lignocellulose. Biotechnol Biofuels. 2016;9:243.

22. Festa S, Coppotelli BM, Madueño L, Loviso CL, Macchi M, Neme Tauil $\mathrm{RM}$, et al. Assigning ecological roles to the populations belonging to a phenanthrene-degrading bacterial consortium using omic approaches. PLOS ONE. 2017;12:1-21.

23. Wong ML, An D, Caffrey SM, Soh J, Dong X, Sensen CW, et al. Roles of thermophiles and fungi in bitumen degradation in mostly cold oil sands outcrops. Appl Environ Microbiol. 2015;81:6825-38.

24. Jiao S, Chen W, Wang E, Wang J, Liu Z, Li Y, et al. Microbial succession in response to pollutants in batch-enrichment culture. Sci Rep. 2016;6:1-11.

25. Nannipieri P, Ascher J, Ceccherini MT, Landi L, Pietramellara G, Renella G. Microbial diversity and soil functions. Eur J Soil Sci. 2017;68:1-26.

26. Loreau $M$, Loreau $M$, Naeem $S$, Naeem S, Inchausti $P$, Inchausti $P$, et al. Biodiversity and ecosystem functioning: current knowledge and future challenges. Science. 2001;294:804-8.

27. Zafra G, Taylor TD, Absalón AE, Cortés-espinosa DV. Comparative metagenomic analysis of PAH degradation in soil by a mixed microbial consortium. J Hazard Mater. 2016:318:702-10.

28. Wang Y, Liu Q, Yan L, Gao Y, Wang Y, Wang W. A novel lignin degradation bacterial consortium for efficient pulping. Bioresour Technol. 2013;139:113-9.

29. Strachan $C R$, Singh R, VanInsberghe D, levdokymenko K, Budwill K, Mohn $W W$, et al. Metagenomic scaffolds enable combinatorial lignin transformation. Proc Natl Acad Sci USA. 2014:111:10143-8.

30. Rocha GJM, Martín C, da Silva VFN, Gómez EO, Gonçalves AR. Mass balance of pilot-scale pretreatment of sugarcane bagasse by steam explosion followed by alkaline delignification. Bioresour Technol. 2012;111:447-52 
31. Miller GL. Use of dinitrosaiicyiic acid reagent for determination of reducing sugar. Anal Chem. 1959;31:426-8.

32. Caporaso JG, Lauber CL, Walters WA, Berg-Lyons D, Huntley J, Fierer $\mathrm{N}$, et al. Ultra-high-throughput microbial community analysis on the Illumina HiSeq and MiSeq platforms. ISME J. 2012;6:1621-4.

33. White TJ, Bruns T, Lee S, Taylor JW. PCR protocols: a guide to methods and applications. Innis MA, Gelfand DH, Sninsky JJ, White TJ, editor. New York: Academic Press, Inc.; 1990

34. Bolger AM, Lohse M, Usadel B. Trimmomatic: a flexible trimmer for Illumina sequence data. Bioinformatics. 2014;30:2114-20.

35. Zhang J, Kobert K, Flouri T, Stamatakis A. PEAR: a fast and accurate Illumina Paired-End reAd mergeR. Bioinformatics. 2014;30:614-20.

36. Edgar RC. UPARSE: highly accurate OTU sequences from microbial amplicon reads. Nat Methods. 2013;10:996.

37. Wang Q, Garrity GM, Tiedje JM, Cole JR. Naïve Bayesian classifier for rapid assignment of rRNA sequences into the new bacterial taxonomy. Appl Environ Microbiol. 2007;73:5261-7.

38. Nilsson RH, Tedersoo L, Ryberg M, Kristiansson E, Hartmann M, Unterseher $M$, et al. A comprehensive, automatically updated fungal ITS sequence dataset for reference-based chimera control in environmental sequencing efforts. Microbes Environ. 2015;30:145-50.

39. Peng Y, Leung HCM, Yiu SM, Chin FYL. IDBA-UD: a de novo assembler for single-cell and metagenomic sequencing data with highly uneven depth. Bioinformatics. 2012:28:1420-8.

40. Mikheenko A, Saveliev V, Gurevich A. MetaQUAST: evaluation of metagenome assemblies. Bioinformatics. 2016;32:1088-90.

41. Zhu W, Lomsadze A, Borodovsky M. Ab initio gene identification in metagenomic sequences. Nucleic Acids Res. 2010;38:1-15.

42. Dominik RL, Mark LB. BlobTools: Interrogation of genome assemblies. F1000Research. 2017;6:1287.

43. Finn RD, Coggill P, Eberhardt RY, Eddy SR, Mistry J, Mitchell AL, et al. The Pfam protein families database: towards a more sustainable future. Nucleic Acids Res. 2016;44:D279-85.

44. Yin Y, Mao X, Yang J, Chen X, Mao F, Xu Y. dbCAN: a web resource for automated carbohydrate-active enzyme annotation. Nucleic Acids Res. 2012;40:W445-51.

45. Huerta-Cepas J, Szklarczyk D, Forslund K, Cook H, Heller D, Walter MC, et al. EGGNOG 4.5: a hierarchical orthology framework with improved functional annotations for eukaryotic, prokaryotic and viral sequences. Nucleic Acids Res. 2016:44:D286-93.

46. Kanehisa M, Sato Y, Morishima K. BlastKOALA and GhostKOALA: kEGG Tools for Functional characterization of genome and metagenome sequences. J Mol Biol. 2016;428:726-31.

47. Alneberg J, Bjarnason BS, de Bruijn I, Schirmer M, Quick J, ljaz UZ, et al. Binning metagenomic contigs by coverage and composition. Nat Methods. 2014;11:1144-6.

48. Langmead B, Salzberg SL. Fast gapped-read alignment with Bowtie. Nat Methods. 2012;2(9):357-9.

49. Parks DH, Imelfort M, Skennerton CT, Hugenholtz P, Tyson GW. CheckM: assessing the quality of microbial genomes recovered from isolates, single cells, and metagenomes. Genome Res. 2015;25:1043-55.

50. Wang Z, Wu M. A phylum-level bacterial phylogenetic marker database. Mol Biol Evol. 2013:30:1258-62.

51. Zerbino DR, Birney E. Velvet: algorithms for de novo short read assembly using de Bruijn graphs. Genome Res. 2008;18:821-9.

52. Boetzer M, Henkel CV, Jansen HJ, Butler D, Pirovano W. Scaffolding preassembled contigs using SSPACE. Bioinformatics. 2011;27:578-9.

53. Walker BJ, Abeel T, Shea T, Priest M, Abouelliel A, Sakthikumar S, et al, Pilon: an integrated tool for comprehensive microbial variant detection and genome assembly improvement. PLOS ONE. 2014;9:e112963.

54. Markowitz VM, Mavromatis K, Ivanova NN, Chen IMA, Chu K, Kyrpides NC. IMG ER: a system for microbial genome annotation expert review and curation. Bioinformatics. 2009;25:2271-8.

55. Darling AE, Tritt A, Eisen JA, Facciotti MT. Mauve assembly metrics. Bioinformatics. 2011;27:2756-7.

56. Kurtz S, Phillippy A, Delcher AL, Smoot M, Shumway M, Antonescu C, et al. Versatile and open software for comparing large genomes. Genome Biol. 2004;5:R12

57. Aslanidis C, De Jong PJ. Ligation-independent cloning of PCR products (LIC-PCR). Nucleic Acids Res. 1990;18:6069-74.
58. Yang W, Tang H, Ni J, Wu Q, Hua D, Tao F, et al. Characterization of two streptomyces enzymes that convert ferulic acid to vanillin. PLoS ONE. 2013;8:e67339.

59. Roessner U, Luedemann A, Brust D, Fiehn O, Linke T, Willmitzer L, et al. Metabolic Profiling Allows Comprehensive Phenotyping of Genetically or Environmentally Modified Plant Systems. Plant Cell. 2001;13:11-29.

60. Weckwerth W, Wenzel K, Fiehn O. Process for the integrated extraction, identification and quantification of metabolites, proteins and RNA to reveal their co-regulation in biochemical networks. Proteomics. 2004;4:78-83.

61. Cuadros-inostroza Á, Caldana C, Redestig H, Kusano M, Lisec J, Peña-cortés $\mathrm{H}$, et al. TargetSearch—a Bioconductor package for the efficient preprocessing of GC-MS metabolite profiling data. BMC Bioinf. 2009;12:1-12.

62. Giavalisco P, Li Y, Matthes A, Eckhardt A, Hubberten $\mathrm{H}$, Hesse H, et al. Elemental formula annotation of polar and lipophilic metab- olites using $13 \mathrm{C}, 15 \mathrm{~N}$ and $34 \mathrm{~S}$ isotope labelling, in combination with high-resolution mass spectrometry. Plant J. 2011;68:364-76.

63. Huege J, Krall L, Steinhauser MC, Giavalisco P, Rippka R, Tandeau De Marsac N, et al. Sample amount alternatives for data adjustment in comparative cyanobacterial metabolomics. Anal Bioanal Chem. 2011;399:3503-17.

64. Dunbar J, Barns SM, Ticknor LO, Kuske CR. Empirical and theoretical bacterial diversity in four arizona soils empirical and theoretical bacterial diversity in four arizona soils. Appl Environ Microbiol. 2002;68:3035-45.

65. Lee $\mathrm{SH}, \mathrm{Ka}$ JO, Cho JC. Members of the phylum acidobacteria are dominant and metabolically active in rhizosphere soil. FEMS Microbiol Lett. 2008;285:263-9.

66. White RA III, Bottos EM, Chowdhury TR, Zucker JD, Brislawn CJ, Nicora CD, et al. Moleculo long-read sequencing facilitates assembly and genomic binning from complex soil metagenomes. mSystems. 2016;1:1-15.

67. Chung SY, Maeda M, Song E, Horikoshi K, Kudo T. A gram-positive polychlorinated biphenyl-degrading bacterium, Rhodococcus erythropolis Strain TA421, isolated from a termite ecosystem. Biosci Biotechnol Biochem. 1994;58:2111-3.

68. Chandra R, Singh S, Krishna Reddy MM, Patel DK, Purohit HJ, Kapley A. Isolation and characterization of bacterial strains Paenibacillus sp. and Bacillus sp. for kraft lignin decolorization from pulp paper mill waste. $J$ Gen Appl Microbiol. 2008;54:399-407.

69. Blanco-Moreno R, Sáez LP, Luque-Almagro VM, Roldán MD, MorenoVivián C. Isolation of bacterial strains able to degrade biphenyl, diphenyl ether and the heat transfer fluid used in thermo-solar plants. N Biotechnol E. 2017;35:35-41.

70. Vandera E, Samiotaki M, Parapouli M, Panayotou G, Koukkou Al. Comparative proteomic analysis of Arthrobacter phenanthrenivorans Sphe3 on phenanthrene, phthalate and glucose. J Proteom. 2015;113:73-89.

71. Weng XY, Sun JY. Biodegradation of free gossypol by a new strain of Candida tropicalis under solid state fermentation: effects of fermentation parameters. Process Biochem. 2006;41:1663-8.

72. Ettayebi K, Errachidi F, Jamai L, Tahri-Jouti MA, Sendide K, Ettayebi M. Biodegradation of polyphenols with immobilized Candida tropicalis under metabolic induction. FEMS Microbiol Lett. 2003;223:215-9.

73. Martorell MM, Pajot HF, de Figueroa LIC. Dye-decolourizing yeasts isolated from Las Yungas rainforest. Dye assimilation and removal used as selection criteria. Int Biodeterior Biodegrad. 2012;66:25-32.

74. Šimek K, Vrba J, Pernthaler J, Posch T, Hartman P, Nedoma J, et al. Morphological and compositional shifts in an experimental bacterial community influenced by protists with contrasting feeding modes. Appl Environ Microbiol. 1997;63:587-95.

75. Jürgens K, Pernthaler J, Schalla S, Amann R. Morphological and compositional changes in a planktonic bacterial community in response to enhanced protozoan grazing morphological and compositional changes in a planktonic bacterial community in response to enhanced protozoan grazing. Appl Environ Microbiol. 1999:65:1241-50.

76. Burlingame R, Chapman PJ. Catabolism of phenylpropionic acid and its 3-hydroxy derivative by Escherichia coli. J Bacteriol. 1983;155:113-21.

77. Jenkinst JR, Cooper RA. Molecular cloning, expression, and analysis of the genes of the homoprotocatechuate catabolic pathway of Escherichia coli Ct. J Bacteriol. 1988;170:5317-24.

78. Zhou NY, Fuenmayor SL, Williams PA. nag genes of Ralstonia (formerly pseudomonas) sp. Strain U2 encoding enzymes for gentisate catabolism. J Bacteriol. 2001;183:700-8. 
79. Campillo T, Renoud S, Kerzaon I, Vial L, Baude J, Gaillard V, et al. Analysis of hydroxycinnamic acid degradation in Agrobacterium fabrum reveals a coenzyme A-dependent, beta-oxidative deacetylation pathway. Appl Environ Microbiol. 2014;80:3341-9.

80. Větrovský T, Steffen KT, Baldrian P. Potential of cometabolic transformation of polysaccharides and lignin in lignocellulose by soil Actinobacteria. PLoS ONE. 2014;9:e89108.

81. Gall DL, Ralph J, Donohue TJ, Noguera DR. A group of sequence-related sphingomonad enzymes catalyzes cleavage of $\beta$-aryl ether linkages in lignin $\beta$-guaiacyl and $\beta$-syringyl ether dimers. Environ Sci Technol. 2014;48:12454-63.

82. Fuchs $\mathrm{G}$, Boll M, Heider J. Microbial degradation of aromatic compounds - from one strategy to four. Nat Rev Microbiol. 2011;9:803-16.

83. Salvachúa D, Karp EM, Nimlos CT, Vardon DR, Beckham GT. Towards lignin consolidated bioprocessing: simultaneous lignin depolymerization and product generation by bacteria. Green Chem R Soc Chem. 2015;17:4951-67.

84. Jeffries TW. Biodegradation of lignin-carbohydrate complexes. Biodegradation 1990;1:163-76.

85. Subramaniyan S, Prema P. Biotechnology of Microbial Xylanases: enzymology. Mol Biol Appl. 2002;22:33-46.

86. Errico C, Jørgensen JO, Krogh KBRM, Spodsberg N. Enzymatic degradation of lignin-carbohydrate complexes (LCCs): model Studies using a fungal glucuronoyl esterase from cerrena unicolor. Biotechnol Bioeng. 2015;112:914-22.

87. Varghese NJ, Mukherjee S, Ivanova N, Konstantinidis T, Mavrommatis K, Kyrpides NC, et al. Microbial species delineation using whole genome sequences. Nucleic Acids Res. 2015;43(14): 6761-71. https://doi. org/10.1093/nar/gkv657

88. Naoto S. Phenolic acids and their cabohydrate esters in rice endosperm cell walls. Phytochemistry. 1984;23:2233-7.

89. Overhage J, Priefert H, Steinbuchel A. Biochemical and genetic analyses of ferulic acid catabolism in Pseudomonas sp. strain HR199. Appl Environ Microbiol. 1999;65:4837-47.

90. Plaggenborg R, Overhage J, Loos A, Archer JAC, Lessard P, Sinskey AJ, et al. Potential of Rhodococcus strains for biotechnological vanillin production from ferulic acid and eugenol. Appl Microbiol Biotechnol. 2006:72:745-55.

91. Achterholt S, Priefert H, Steinbüchel A. Identification of Amycolatopsis sp. strain HR167 genes, involved in the bioconversion of ferulic acid to vanillin. Appl Microbiol Biotecnhol. 2000;103:799-807.

92. Gasson MJ, Kitamura Y, Mclauchlan WR, Narbad A, Parr AJ, Parsons ELH, et al. Metabolism of ferulic acid to vanillin. A bacterial gene of the enoylSCOA hydratase/isomerase superfamily encodes an enzyme for the hydration and cleavage of a hydroxycinnamic acid SCoA thioester. J Biol Chem. 1998;273:4163-70

93. Calisti C, Ficca AG, Barghini P, Ruzzi M. Regulation of ferulic catabolic genes in Pseudomonas fluorescens BF13: involvement of a MarR family regulator. Appl Microbiol Biotecnhol. 2008;80:475-83.

94. Reizer A, Deutscher J, Saier MH. Analysis of the giuconate (gnt) operon of Bacillus subtilis. Mol Microbiol. 1991;5:1081-9.

\section{Submit your next manuscript to BioMed Central and we will help you at every step:}

- We accept pre-submission inquiries

- Our selector tool helps you to find the most relevant journal

- We provide round the clock customer support

- Convenient online submission

- Thorough peer review

- Inclusion in PubMed and all major indexing services

- Maximum visibility for your research

Submit your manuscript at www.biomedcentral com/submit

O Biomed Central 